\title{
Quasiparticle Dynamics in the Vicinity of Metal-Insulator Phase Transition in $\mathrm{Na}_{x} \mathrm{CoO}_{2}$
}

\author{
D. Qian, ${ }^{1}$ L. Wray, ${ }^{1}$ D. Hsieh, ${ }^{1}$ D. Wu, ${ }^{2}$ J. L. Luo, ${ }^{2}$ N. L. Wang, ${ }^{2}$ A. Kuprin, ${ }^{3}$ A. Fedorov, ${ }^{3}$ \\ R. J. Cava, ${ }^{4}$ L. Viciu, ${ }^{4}$ and M.Z. Hasan ${ }^{1,5, *}$ \\ ${ }^{1}$ Department of Physics, Joseph Henry Laboratories of Physics, Princeton University, Princeton, New Jersey 08544, USA \\ ${ }^{2}$ Beijing National Laboratory for Condensed Matter Physics, Chinese Academy of Sciences, Beijing 100080, China \\ ${ }^{3}$ Advanced Light Source, Lawrence Berkeley Laboratory, Berkeley, California 94305, USA \\ ${ }^{4}$ Department of Chemistry, Princeton University, Princeton, New Jersey 08544, USA \\ ${ }^{5}$ Princeton Center for Complex Materials, Princeton University, Princeton, New Jersey 08544, USA
}

(Received 9 October 2005; published 2 February 2006)

\begin{abstract}
Layered cobaltates embody novel realizations of correlated matter on a spin-1/2 triangular lattice. We report a high-resolution systematic photoemission study of the insulating cobaltates. The observation of a single-particle gap opening and band folding provides direct evidence of anisotropic particle-hole instability on the Fermi surface due to its unique topology. Overlap of the measured Fermi surface is observed with the $\sqrt{3} \times \sqrt{3}$ charge-order Brillouin zone near $x=1 / 3$ but not at $x=1 / 2$ where the insulating transition is actually observed. Unlike conventional density waves, charge stripes, or band insulators, the onset of the gap depends on the quasiparticle's quantum coherence which is found to occur well below the disorder-order symmetry breaking temperature of the crystal (the first known example of its kind).
\end{abstract}

DOI: 10.1103/PhysRevLett.96.046407

Strong electron-electron interaction (Mott physics) is known to be the origin of metal to insulator transitions in many oxides [1]. Such systems exhibit strong quantum effects if electron transport occurs in lower dimensions and the underlying band is half filled (spin 1/2). Recently discovered $\mathrm{Na}_{x} \mathrm{CoO}_{2}$ is the first realization of a spin- $1 / 2$ doped Mott insulator on a triangular lattice [2-4]. This system exhibits not only superconductivity [5] and spindependent thermopower [6] but also antiferromagnetism and spin-density-wave phases [2] as well as an unusual metal-insulator transition (Fig. 1) [2-4,7,8]. Most Mott systems exhibit insulating phases while doped near the natural commensurate values of the underlying lattice [1]. Surprisingly, the insulating state in cobaltates is observed near the $x=1 / 2$ doping, which is not a natural commensurate value $(x=1 / 3$ or $2 / 3)$ of the triangular lattice. This remains an unsolved issue to date. In this Letter, we probe the microscopic electron dynamics in the vicinity of the metal-insulator phase transition in $\mathrm{Na}_{x} \mathrm{CoO}_{2}$, which reveals that the insulating state in cobaltates is due to an anisotropic particle-hole instability on the Fermi surface resulting from its unique momentum-space topology with respect to the $\mathrm{Na}^{+}$charge order. However, unlike the conventional charge-density waves, the onset of the insulating gap (order parameter) is related to the energy scale of the emergence of the quasiparticle's quantum coherence instead of the disorder to order symmetry breaking temperature of the crystal, which is the first known example of its kind.

Electron [2,3], neutron [4], infrared (IR) [7], Shubnikov-de Haas (SdH) [9], and NMR measurements [10] have been used to study the insulating state. However, angle-resolved photoemission spectroscopy (ARPES) studies have so far been carried out only on the metallic
PACS numbers: 71.20.Be, 71.27.+a, 71.30.+h, 73.20.At

cobaltates [11]. In this Letter, we report a single-particle study of the cobaltate insulator for the first time. High quality single crystals of $\mathrm{Na}_{x} \mathrm{CoO}_{2}$ and $\mathrm{K}_{1 / 2} \mathrm{CoO}_{2}$ and nearby dopings were grown by the floating zone and flux methods, respectively. Na concentration near 0.5 (0.49 to 0.51 ) was achieved by postgrowth deintercalation, whereas $\mathrm{K}$ samples could be naturally grown with $x=0.5$ ( 0.47 to $0.53)$ doping. Insulating states with two characteristic resistivity upturns around 50 and $25 \mathrm{~K}$ in Na samples and 60 and $20 \mathrm{~K}$ in $\mathrm{K}$ samples were observed. No surface state was observed. Spectroscopic measurements were performed at the Advanced Light Source. The data were collected with He I $(21.2 \mathrm{eV}), 30$ or $60 \mathrm{eV}$ photons with better than 8,12 , or $10 \mathrm{meV}$ energy resolution and an angular resolution better than $2 \%$ of the Brillouin zone (BZ) at beam lines 12.0.1 and 10.0.1 using Scienta analyzers with chamber pressures better than $4 \times 10^{-11}$ torr.

No measurable sign of sample charging was observed down to $10 \mathrm{~K}$. No appreciable loss of $\mathrm{Na} 2 p$ (or K $3 p$ ) peak intensity was observed within the probe depth of ARPES and within a few hours of cleaving in UHV. Such care has been quite crucial in observing the energy gap since a change of doping $(>3 \%)$ or aging turns the sample into a metal with a large Fermi surface (FS). Measured FS area further provided an internal check for changes in effective doping on the surface.

Figures 2(a)-2(c) show the quasiparticle dispersion as the system approaches the insulating phase from either side of the doping phase diagram (namely, $x=0.3, x=0.5$, and $x=0.55$ ) within $200 \mathrm{meV}$ of the electron binding energies at $20 \mathrm{~K}$. Only in $x=0.5$ samples is spectral weight suppression observed at low temperature. The gap behavior is seen in the energy distribution curves in Figs. 2(d) $-2(\mathrm{~g})$. The $x=0.5$ samples show a clear pull- 

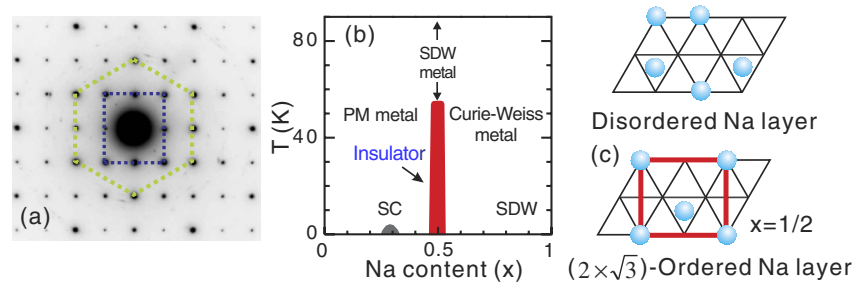

FIG. 1 (color online). Phase transitions in $\mathrm{Na}_{x} \mathrm{CoO}_{2}$ : (a) Electron diffraction image along the [001] axis. The rectangular supercell is observed due to the $\mathrm{Na}^{+}$charge ordering (Wigner crystallization). (b) Phase diagram of $\mathrm{Na}_{x} \mathrm{CoO}_{2}$ [2]. PM stands for paramagnetic. Metal-insulator phase transitions are observed in doping near $x=1 / 2$ where (c) the Na layer undergoes a disorder to order transition above room temperature [2-4].

back of the quasiparticle along the high symmetry cut $\Gamma \rightarrow$ $K$. A spectral gap is also observed along $\Gamma \rightarrow M$, but the folding behavior is less prominent. These quasiparticles emerge from the $a_{1 g}$-like states. We have carefully monitored changes at the $e_{g^{\prime}}$ bands under $S$ geometry, as they are predicted to be involved in the ordering transition $[12,13]$. The $e_{g^{\prime}}$ band is found to be fully gapped and hardly showed any temperature or doping dependence [Fig. 2(h)]. Therefore we conclude that the insulating gap opens by the destruction of the $a_{1 g}$ Fermi surface only [Fig. 2(i)].

Figure 3(a) shows integrated (momentum window of $0.06 \AA 4 \%$ of the BZ) low-energy spectra near the folding point along $\Gamma \rightarrow M, \Gamma \rightarrow K$, and cuts in between. The data are compared to a spectrum from a nearby metallic sample $(x=0.55)$. The gap energy $\Delta$ varies from 6 to $11 \mathrm{meV}$. Angular distribution of the gap is plotted in Fig. 3(b). The gap tends to be larger along $\Gamma \rightarrow K$ cuts.

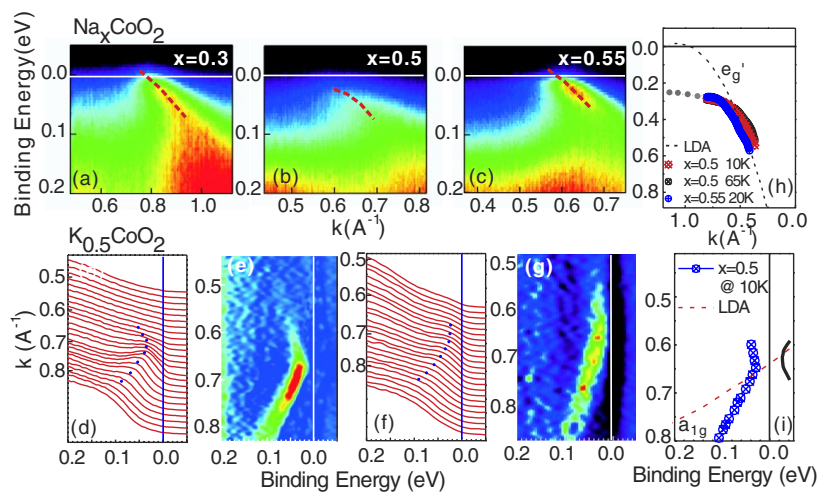

FIG. 2 (color online). Quasiparticle dynamics: Single-particle removal spectra of samples with doping at (a) $x=0.3$, (b) $x=0.5$, and (c) $x=0.55$. (d) and (e) show spectra and color images along $\Gamma \rightarrow K$, while (f) and (g) are spectra measured along $\Gamma \rightarrow M$. (h) Doping and temperature dependence of $e_{g^{\prime}}$-band dispersions in the vicinity of $x=0.5$ which show no changes. (i) Experimental band dispersion is compared with local-density approximation (LDA) theory (dotted line) [24]. The measured band is less dispersive. It exhibits folding and a gap opens at the Fermi level.
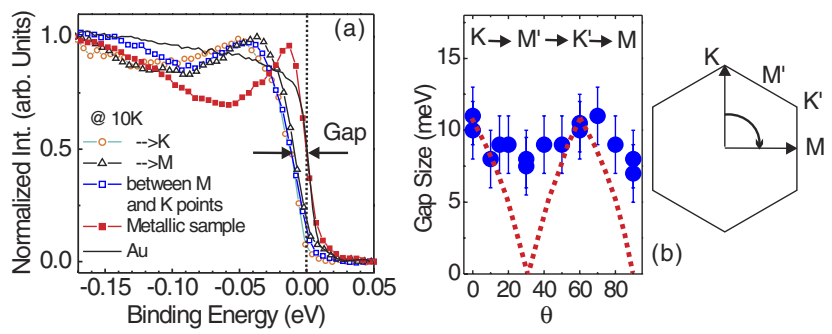

FIG. 3 (color online). Gap anisotropy: (a) High energyresolution ( $k$ integrated on $4 \% \mathrm{BZ}$ ) spectra of $\mathrm{Na}_{0.5} \mathrm{CoO}_{2}$ measured at $10 \mathrm{~K}$ near the folding points along different $k$-space cuts. Comparison of the spectra with the Fermi edge of $\mathrm{Au}$ and metallic $\mathrm{Na}_{0.55} \mathrm{CoO}_{2}$ demonstrates the gap in $\mathrm{Na}_{0.5} \mathrm{CoO}_{2}$. (b) $k$ dependence of quasiparticle (leading-edge) gap in $\mathrm{K}_{0.5} \mathrm{CoO}_{2}$ measured at $10 \mathrm{~K}$. Open circles are the expected $k$ dependence of the gap in case where nesting occurring only along $\Gamma \rightarrow K$ line. The $k$-space angle $\theta$ is defined from the $\Gamma \rightarrow K$ to the $\Gamma \rightarrow$ $M$ line.

A hexagonal Fermi surface is observed in nearby dopings $(x=0.3$ or 0.55$)$ at low temperatures, but no band folding is seen. In $x=0.5$ the hexagonal Fermi surface is found to be destroyed at low temperatures by the band folding. Figure 4(a) shows the $n(k)$ map generated with an integration window of $20 \mathrm{meV}$ ( $>$ gap size). This shows the topology of the "Fermi surface" around the onset of gap opening. This Fermi surface retains a hexagonal character except that its volume has changed according to
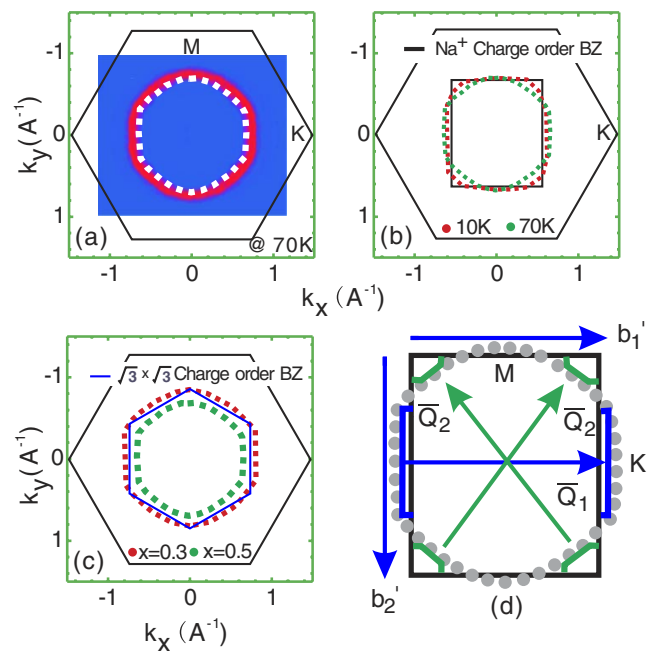

FIG. 4 (color online). Particle-hole instabilities on the Fermi surface: Fermi surface reconstruction: $\mathrm{Na}^{+}$charge order vs $\sqrt{3} \times \sqrt{3}$ Co charge order. (a) Momentum distribution of quasiparticles (two-color image), $n(k)$, within $20 \mathrm{meV}$ of Fermi energy. The white dots reflect the FS topology. (b) Temperature dependence of the "FS topology" in $x=1 / 2$ samples. The solid rectangle is the $\mathrm{BZ}$ of Na supercell. Measured FS topology almost coincides with the Na supercell. (c) The $\sqrt{3} \times \sqrt{3}$ Co charge-order BZ (solid hexagon) has no overlap with $x=0.5 \mathrm{FS}$ but coincides with $x=0.3$ (superconducting doping) FS. (d) Reconstructed FS contours based on experimental folding behavior, which is consistent with the geometry of the $\mathrm{Na}$ supercell defined by $\overrightarrow{b_{1}^{\prime}}$ and $\overrightarrow{b_{2}^{\prime}}$. 
doping. The size of the measured FS at $x=1 / 2$ doping is found to be special when the $\mathrm{Na}^{+}$superlattice is considered. In reciprocal space, the primary vector $\mathrm{G}$ in hexagonal cell is $2.6 \AA^{-1}$. This is much larger than any possible $2 k_{f}$ in any doping over the phase diagram. However, considering the $\mathrm{Na}^{+}$lattice $(2 \times \sqrt{3}$ supercell BZ), the shortest vectors G's are 1.1 and $1.3 \AA^{-1}$, which is close to the measured value of average $2 k_{f}$ for doping in the neighborhood of 0.5 [Fig. 4(b)]. Our results also show that the $\sqrt{3} \times$ $\sqrt{3}$ long-range charge-order BZ [8] has no overlap with $x=0.5$ FS [Fig. 4(c)], hence likely not related to the insulating behavior at this particular doping. The Na superstructure imposes a twofold symmetry on the FS. The $\mathrm{Na}^{+}$ ionic potential likely enhances nesting conditions $\left(\vec{Q}_{1} \|\right.$ $\vec{b}_{1}^{\prime}, \vec{Q}_{2}$ ) along the $\Gamma \rightarrow K / K^{\prime}$ cuts [Fig. 4(d)]. This should then be reflected in the gap and folding behavior. We also consider the domains of the supercell. With a fixed hexagonal matrix, there are 3 possible domains for the $\mathrm{Na}^{+}$ supercell. The domains mix $K$ and $K^{\prime}$ as well as $M$ and $M^{\prime}$ but not $M$ with $K$ or $M^{\prime}$ with $K^{\prime}$. Therefore, even in case of complete domain mixing one would expect clear, distinct, and different folding and gap behaviors to survive along $\Gamma \rightarrow M / M^{\prime}$ and $\Gamma \rightarrow K / K^{\prime}$, consistent with our observation. In a conventional density wave picture [14,15], gap size is large along the longest straight sections of the FS. Nesting possibility along $\Gamma \rightarrow K / K^{\prime}$ suggests that the gap would be larger and the band will exhibit stronger folding behavior since ARPES intensity of folding is proportional to nesting strength [15]. It is likely that the effect of the sodium supercell is to flatten out the FS also along $\Gamma \rightarrow M$, which then leads to a gap. A weaker gap would be expected in such a scenario compared to what was actually observed along $M$. We note that $\mathrm{Na}_{1 / 2} \mathrm{CoO}_{2}$ is not a conventional charge-density wave (CDW) material like $\mathrm{NbSe}_{3}$ (band metal) is and other effects (finite correlation gap, etc.) are likely at play near the $M$ point.

Figures 5(a) and 5(b) show the temperature dependence of resistivity and quasiparticle spectral weight. Quasiparticles get sharper and the energy gap increases as the temperature is lowered. Figure 5(c) shows the temperature dependence of the charge gap. Temperature dependence of gap viewed as a BCS-like order parameter $[\Delta(T)]$ of the transition suggests that its amplitude vanishes beyond 75$80 \mathrm{~K}$. This is lower than the spin-density wave (SDW) transition $(\sim 88 \mathrm{~K})$ and suggests the gap to be associated with the insulating transitions [2]. Upon further raising the temperature, both the gap size and the spectral weight decrease (beyond thermal broadening). However, the spectral weight does not completely vanish even after the gap has disappeared. Such decrease with rising temperatures is also seen in nearby metallic dopings [Fig. 5(d)]. ARPES studies have shown that in the coherent transport regime quasiparticles gain significant weight due to enhanced or effective out-of-plane coupling or $c$-axis coherence [16].

Our observation of quasiparticle weight mainly below $150 \mathrm{~K}$ in $x=1 / 2$ insulators suggests that even if a Na/K layer is ordered well above room temperature $(\sim 350 \mathrm{~K})$ the cobalt states are affected by the Na-ion potential after quasiparticles form or gain weight. Therefore, if some approximate nesting is to be operative it can take place only at a similar or lower temperature scale following the growth of quasiparticles. We observe such correlation between the charge gap and the quasiparticle coherence in insulating cobaltates (Fig. 5). Similar behavior is seen in cuprate superconductors (SCs) where a SC gap opens shortly after the onset of (coherent) quasiparticles, which has been ascribed to be due to out-of-plane coupling [17]. In case of cobaltates, instead, it leads to a charge-gap opening as seen in our data, which is a competing instability with superconductivity. This also argues that the gap we observe is not as one in a band insulator (Slater-type) by the electron count in the new unit cell otherwise it would have appeared at the onset of $\mathrm{Na}$ order and "FS" would have deformed to exactly coincide with the supercell BZ boundary leading to a zero count as in a particle number vanishing phase transitions. This does not happen even down to $10 \mathrm{~K}$, so there is no adiabatic continuity to a Slater band insulator phase. It is possible that one of the nesting or modulation vectors $\left(\vec{Q}_{1}\right)$ becomes operative (longer straight section, hence stronger coupling, stronger folding) around $60 \mathrm{~K}$ (first upturn in resistivity [Fig. 5(a)]), which nests a good part of the FS but not completely, whereas at lower temperatures near $20 \mathrm{~K}$ both vectors $\left(\vec{Q}_{1}\right.$, $\vec{Q}_{2}$, possibly also along $M$ ) are in effect gapping most of the FS thus leading to resistivity divergence. Such behavior is consistent with nuclear resonance data [10].

Density wave modulations in matter form via several different mechanisms. Singularity in the density of states helps realize a density wave state [18]. In a triangular system such singularities in the density of states are expected in case of large and positive $t$ [19]. However, $\mathrm{Na}_{1 / 2} \mathrm{CoO}_{2}$ is in the opposite limit, namely, with a negative sign of hopping ( $t<0$, holelike band) with a small $t_{\mathrm{eff}}$ $(\sim 16 \mathrm{meV} \sim$ bandwidth $(0.15 \mathrm{eV}) / 9)$. A more ubiquitous mechanism for developing a density wave modulation is via FS instability. This is the case for $\mathrm{NbSe}_{3}$ and other 1D metals that exhibit conventional behavior [20]. In most two dimensional materials (2H-NbSe 2 , etc. [21]) nesting is never complete and the FS is partially gapped and the system remains fairly metallic (no insulating divergence). Given the transition temperature $(\sim 60 \mathrm{~K})$ in cobaltates, the gap size $(10 \mathrm{meV})$ is rather soft compared to many other systems where $\Delta \gg k_{B} T_{c}$. The gap closes much faster in cobaltates than it is seen in most CDW systems [20,21]. The coherence length of the density wave modulation in cobaltate, based on our data [ $v_{f} \sim 0.5 \mathrm{eV} \AA$ (Fig. 2): $\xi \sim$ $\left.\hbar v f / \Delta \sim 10^{2} \AA\right]$ is less than the correlation length of $\mathrm{Na}$ charge order [4] consistent with a density wave induced on the Co plane. Another common mechanism for generating a spectral gap is via valence charge order as argued in [24]. Many oxides indeed exhibit charge-ordering (stripes) due to electron-electron and electron-lattice interactions 

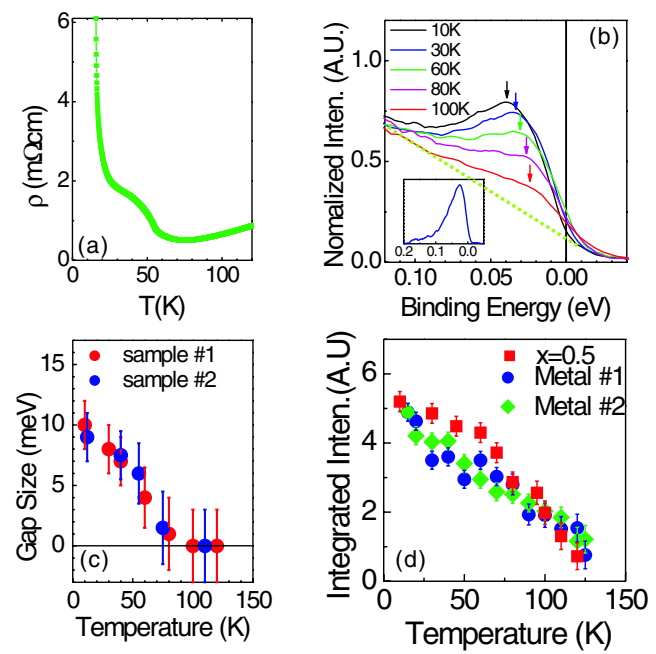

FIG. 5 (color online). Charge-gap amplitude and quasiparticle coherence: Temperature dependence of (a) in-plane dc resistivity, (b) quasiparticle signal along $\Gamma \rightarrow K$ cut. Inset shows the quasiparticle after linear background subtraction. (c) Evolution of leading-edge gap with temperature. (d) The integrated spectral weight of quasiparticle (coherence) in insulating doping ( $x=$ $0.5)$ is compared with the metallic dopings $(x=0.6-0.7)$ as a function of temperature.

[1]. Such order typically leads to a gap often known as weak correlation gap or pseudogap [1]. No overlap (intersection) of our measured Fermi surface is observed with the $\sqrt{3} \times \sqrt{3}$ cobalt long-range charge-order Brillouin zone. However, a combination of FS instability and correlation effects can lead to a weakly k-dependent (pseudo)gap or correlation gap in manganites in the case of fluctuating charge order [22]. The lack of valence disproportion occurring at the transition [10] has been used to argue for a fluctuating mechanism via intersite Coulomb (V) which likely sets the gap amplitude [23]. This can lead to a finite correlation gap (then the weak $k$ modulation of the gap reflects the fine FS topological instability effects) in cobaltates. Irrespective of the mechanism of spectral gapping, our results are consistent with IR [7] and $\mathrm{SdH}$ measurements [9] where it is argued that almost entire FS vanishes at the transition except extremely small leftover pockets $(0.25 \%$ of the hex-BZ). Such a scenario is consistent with gap and band folding we observe. The reconstructed corners [Fig. 4(d)] are not likely resolved due to finite resolution and weak folding strengths [SdH [9] does not report the exact $k$-space locations of the pockets; hence a detailed comparison is not possible with the dispersion folding ( $E$ vs $k$ ) and gap we report by ARPES.]

In conclusion, we demonstrate that the coupling of quasiparticle states with the crystallized $\mathrm{Na}$ layer indeed leads to an intrinsic quantum many-body (correlated) insulatinglike low-energy state with a weakly $k$-modulated gap $(\sim 6-11 \mathrm{meV})$ via particle-hole instability on the Fermi surface. Kinematic overlap of the measured Fermi surface is observed with the $\sqrt{3} \times \sqrt{3}$ cobalt charge-order Brillouin zone near $x=1 / 3$ but not at $x=1 / 2$. The gap opening and quasiparticle signals (coherence) set in at a similar temperature scale which is much smaller than the $\mathrm{Na} / \mathrm{K}$ charge-order scale and exhibit spectral redistributions over large energy scales in doping and temperature. A comprehensive many-body theory requires going beyond a conventional one and needs to consider the quasiparticle coherence effects, selective coupling to the superpotential as well as longer-range Coulomb correlations in driving this novel phase transition. Further details of the correlated charge dynamics could be obtained by inelastic x-ray scattering [25]

We thank M. Lee for the resistivity measurements on the samples. We gratefully acknowledge D. A. Huse, M. R. Beasley, D. H. Lee, M. Lee, P. A. Lee, N.P. Ong, P. Phillips, S. Shastry, and S.A. Kivelson for discussions. This work is partially supported through the NSFMRSEC (DMR-0213706), DOE Grants No. DE-FG0205ER46200 and No. DE-FG02-98-ER45706, and NSFC (No. 10574158). ALS is supported by DOE/BES-DEAC03-76SF00098.

*Electronic address: mzhasan@ princeton.edu

[1] M. Imada, A. Fujimori, and Y. Tokura, Rev. Mod. Phys. 70, 1039 (1998).

[2] M. L. Foo et al., Phys. Rev. Lett. 92, 247001 (2004).

[3] H. W. Zandbergen et al., Phys. Rev. B 70, 024101 (2004).

[4] Q. Huang et al., J. Phys. Condens. Matter 16, 5803 (2004).

[5] K. Takada et al., Nature (London) 422, 53 (2003).

[6] Y. Wang et al., Nature (London) 423, 425 (2003).

[7] N. L. Wang et al., Phys. Rev. Lett. 93, 147403 (2004).

[8] O. I. Motrunich and P. A. Lee, Phys. Rev. B 69, 214516 (2004); 70, 024514 (2004).

[9] L. Balicas et al., Phys. Rev. Lett. 94, 236402 (2005).

[10] M. Yokoi et al., cond-mat/0506220; J. Bobroff et al., cond-mat/0507514.

[11] M.Z. Hasan et al., Phys. Rev. Lett. 92, 246402 (2004); H. B. Yang et al., ibid. 95, 146401 (2005).

[12] H. Ishida, M.D. Johannes, and A. Liebsch, Phys. Rev. Lett. 94, 196401 (2005).

[13] M. D. Johannes et al., Phys. Rev. Lett. 93, 097005 (2004).

[14] G. Gruner, Density Waves in Solids (Addison-Wesley, Reading, 1994).

[15] J. Voit et al., Science 290, 501 (2000).

[16] A. Kaminski et al., Phys. Rev. Lett. 90, 207003 (2003).

[17] P. W. Anderson, The Theory of Superconductivity in High $T_{c}$ Cuprates (Princeton University Press, Princeton, NJ, 1997).

[18] T. M. Rice and G. K. Scott, Phys. Rev. Lett. 35, 120 (1975).

[19] B. Kumar and B. S. Shastry, Phys. Rev. B 68, 104508 (2003).

[20] J. Schafer et al., Phys. Rev. Lett. 87, 196403 (2001).

[21] G.-H. Gweon et al., Phys. Rev. Lett. 81, 886 (1998).

[22] Y.-D. Chuang et al., Science 292, 1509 (2001).

[23] T. P. Choy et al., cond-mat/0502164.

[24] D. J. Singh, Phys. Rev. B 61, 13397 (2000).

[25] M.Z. Hasan et al., Phys. Rev. Lett. 88, 177403 (2002); Science 288, 1811 (2000). 